\title{
EchoGéo
}

$52 \mid 2020$

Ho Chi Minh Ville, terrain de jeu(x) métropolitain(s)

\section{L'immobilier residentiel : principal moteur de la fabrique urbaine de Ho Chi Minh Ville}

Discussion à partir du cas de l'aménagement du district 2 et de la Nouvelle Zone Urbaine de Thủ Thiêm

Clément Musil, Danielle Labbé et Vu Ngoc Khanh

\section{CpenEdition}

Journals

Édition électronique

URL : https://journals.openedition.org/echogeo/19633

DOI : $10.4000 /$ echogeo.19633

ISSN : 1963-1197

Éditeur

Pôle de recherche pour l'organisation et la diffusion de l'information géographique (CNRS UMR 8586)

Référence électronique

Clément Musil, Danielle Labbé et Vu Ngoc Khanh, « L'immobilier residentiel : principal moteur de la fabrique urbaine de Ho Chi Minh Ville», EchoGéo [En ligne], 52 | 2020, mis en ligne le 30 juillet 2020, consulté le 10 août 2021. URL : http://journals.openedition.org/echogeo/19633 ; DOI : https://doi.org/ 10.4000/echogeo.19633

Ce document a été généré automatiquement le 10 août 2021.

EchoGéo est mis à disposition selon les termes de la licence Creative Commons Attribution - Pas d'Utilisation Commerciale - Pas de Modification 4.0 International (CC BY-NC-ND) 


\section{L'immobilier residentiel : principal moteur de la fabrique urbaine de Ho Chi Minh Ville}

Discussion à partir du cas de l'aménagement du district 2 et de la Nouvelle Zone Urbaine de Thủ Thiêm

Clément Musil, Danielle Labbé et Vu Ngoc Khanh

Les travaux de recherche présentés dans cet article ont été soutenus par le Conseil de recherches en sciences humaines (CRSH) du Canada (subvention \#430-2016-00144), par la Chaire de recherche du Canada en urbanisation durable dans le Sud Global et par l'Observatoire Ivanhoé Cambridge du développement urbain et immobilier de l'Université de Montréal.

1 Dans les pays émergents, et ceux dits à "revenus intermédiaires", le financement des infrastructures et des services de base destinés aux territoires en cours d'urbanisation est une préoccupation majeure des gouvernements locaux en raison de ressources financières publiques limitées (Floater et al., 2017; Chatry, 2019). C'est le cas de Ho Chi Minh Ville, une métropole qui, malgré sa très forte croissance économique des dernières décennies, peine à financer les infrastructures dont elle a besoin pour aménager son territoire et encadrer sa croissance urbaine (World Bank, 2019).

Depuis les réformes du Đổi Mới (Renouveau) ${ }^{1}$, la métropole du sud du Vietnam peut compter sur des revenus propres pour aménager son territoire. Elle dispose d'un budget transféré par l'État central et de possibilités d'emprunts, ainsi que d'aides financières provenant d'institutions financières internationales (Musil et Perset, 2015). Ces revenus sont néanmoins insuffisants pour réaliser les plans d'aménagement ambitieux adoptés par la métropole ${ }^{2}$. D'autant plus que l'accès aux fonds d'Aide Publique au Développement s'est resserré depuis l'entrée en 2010 du Vietnam dans la catégorie des "pays à revenus intermédiaires ». Ho Chi Minh Ville, comme les autres villes du pays, cherche dès lors à diversifier ses sources de financement.

3 C'est dans ce contexte que la métropole teste depuis quelques années de nouvelles approches de production des infrastructures urbaines, et notamment un dispositif de financement basé sur le foncier (land-based financing) appelé terres-contre- 
infrastructure (land-for-infrastructure) (Labbé et Musil, 2014; Nguyen et al., 2018). S'apparentant à un partenariat public-privé, ce dispositif permet à une municipalité de réaffecter à des investisseurs les droits d'usage de terrains et de les autoriser à les aménager à des fins lucratives en contrepartie de la réalisation d'infrastructures. La production urbaine repose alors sur des arrangements entre l'autorité planificatrice métropolitaine et divers acteurs du secteur privé (dont les anciennes sociétés étatiques) porteur de capitaux en partie issus de leurs activités sur les marchés foncier et immobilier. Cette situation confère aux grandes firmes privées (et aux anciennes sociétés étatiques) impliquées dans les secteurs de la construction et de l'immobilier une place de première importance tant dans le financement que dans la réalisation des projets d'aménagement.

4 Cet article explore le contexte au sein duquel prend place cet accroissement du rôle des acteurs privés de la promotion immobilière dans la production des villes vietnamiennes et ses effets sur la fabrique des nouveaux territoires urbains. Il s'appuie sur le cas du district 2 et de la Nouvelle Zone Urbaine (NZU) de Thủ Thiêm, une zone dont l'aménagement, approuvé au début des années 1990, visait à créer un nouveau centre urbain multifonctionnel (illustrations 1 et 2) (Harms, 2016). Combiné à une analyse critique de l'évolution des politiques publiques du Vietnam en matière d'aménagement du territoire, ce cas suggère que, à l'image de plusieurs autres pays de la région, le rôle accru des acteurs de la promotion immobilière est facilité par ce que Shatkin (2016; 2017) nomme un "virage immobilier des politiques publiques et de la planification ". Ce virage consiste en une réorientation progressive des politiques de développement urbain par les États dans le but de générer des revenus budgétaires, de renforcer les liens avec les puissants acteurs économiques qui les soutiennent et de stimuler la croissance économique. Reflétant la situation observée par Shatkin à travers l'Asie en développement, le virage immobilier vietnamien repose sur des stratégies de monétisation du foncier, entendu ici comme l'utilisation par l'État de ses pouvoirs pour capturer les revenus de la valorisation foncière ou pour permettre à ses alliés du secteur économique (privé comme public) de le faire. La montée en puissance des acteurs de la promotion immobilière et leur implication dans le dispositif terrescontre-infrastructure esquissé plus haut s'inscrivent dans ce contexte. 
Illustration 1 - Le district 2 et les principaux grands projets d'aménagement publics dans les districts centraux de Ho Chi Minh Ville

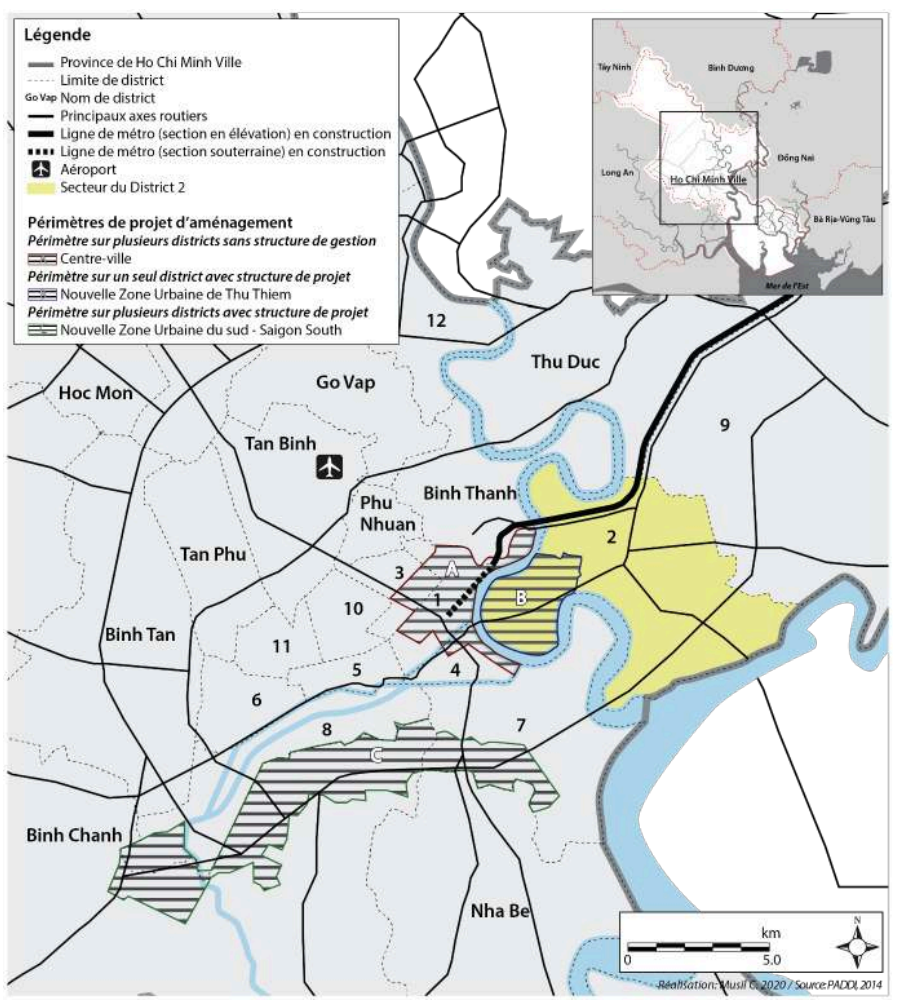

Auteurs : Musil, Labbé et Vu, 2020)

Illustration 2 - La presqu'ile de Thủ Thiêm

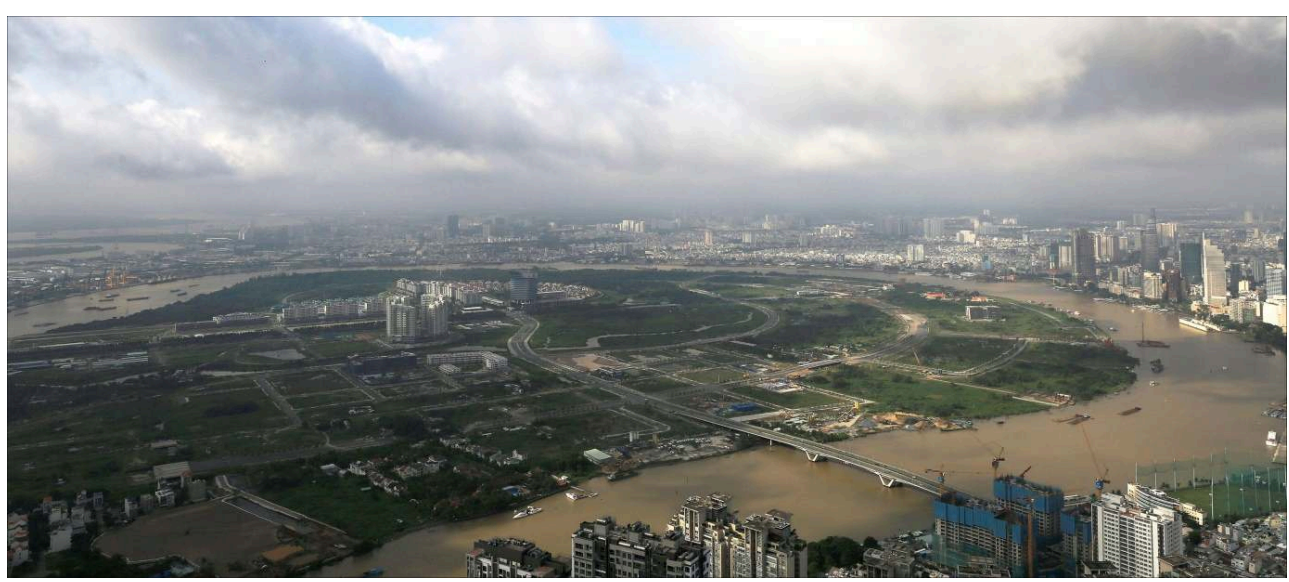

Approuvé en 1996, la planification de cette Nouvelle Zone Urbaine prévoit de créer un nouveau centre tertiaire et de nouveaux quartiers résidentiels pour Ho Chi Minh Ville sur les bords de la rivière Saigon face au centre-ville historique.

Auteur : Musil, 2018.

Dans la foulée du virage immobilier en cours, les politiques publiques et de la planification au Vietnam tentent de conjuguer aménagement de grands ensembles à dominante résidentielle (telle la NZU de Thủ Thiêm) et production d'infrastructures publiques via le dispositif terres-contre-infrastructure. Les effets et l'efficacité de cette stratégie sont discutables. Il apparait que le développement immobilier commercial précède la réalisation des infrastructures pourtant indispensables pour structurer et 
organiser le développement urbain. Les pouvoirs publics peinent à produire suffisamment d'équipements de qualité et à faire respecter à leurs partenaires économiques les délais de livraison. Le contexte politique explique en partie cet échec. L'élaboration et la mise en œuvre des politiques de développement urbain prennent place dans un contexte marqué à la fois par une planification dirigiste, une forte interpénétration des activités et des intérêts économiques des élites économiques et politiques et une opacité des marchés et de la prise de décision publique propices aux arrangements et à la corruption. Le cas du district 2 illustre et révèle cette situation puisque de larges pans de la production urbaine sont sous-traités à un groupe d'acteurs tirant de larges bénéfices de leurs activités immobilières.

6 Cette contribution s'appuie sur l'analyse critique de documents de politiques publiques et de textes réglementaires ainsi que des opérations immobilières dans le district 2 de Ho Chi Minh Ville. L'analyse se base également sur douzaine d'entretiens conduits avec des informateurs-clés du secteur de l'immobilier à Ho Chi Minh Ville en 2017 et 2018. L'analyse complémentaire de rapports de recherche et d'articles de presse a permis de dresser un état des lieux de l'activité immobilière dans le district 2 .

Dans ce qui suit, la première partie de cet article revient sur l'évolution des principales orientations et politiques en matière d'aménagement du territoire via lesquelles l'Étatparti vietnamien a facilité l'implication du secteur privé dans la production urbaine au Vietnam de manière générale et à Ho Chi Minh Ville en particulier. Par le prisme de l'aménagement du district 2 et de la NZU de Thủ Thiêm, une seconde partie rend compte des dynamiques du marché immobilier, de ses caractéristiques et de son effervescence actuelle. Une troisième partie analyse les effets induits par l'utilisation des outils de land-based financing en pointant la nature des conflits qui émergent. En conclusion est discutée la durabilité du modèle de financement désormais à l'œuvre impliquant les acteurs de la promotion immobilière ainsi que le rôle de régulation joué par les autorités politiques.

\section{Réorienter les politiques publiques et de planification urbaine pour stimuler le développement des métropoles vietnamiennes}

Saisir le rôle désormais incontournable des acteurs de la promotion immobilière dans l'aménagement des villes vietnamiennes, et en particulier à Ho Chi Minh Ville, implique de revenir sur d'importants changements d'orientation pris par l'État-parti vietnamien dans le cadre des réformes du Đổi Mới. Si la période d'économie administrée a été caractérisée par la conduite de politiques « anti-urbaines » (Murray et Szelenyi, 1988), le Đổi Mới marque au contraire un repositionnement des villes et de l'urbanisation au centre de la stratégie de développement économique nationale (Gibert-Flutre, 2018; Goldblum, 2017, p. 331). L'urbanisation s'intensifie de façon spontanée dès le début des années 1990 suite à l'assouplissement du contrôle étatique sur les activités économiques privées et sur la migration interne. Suivent d'importantes transformations du cadre législatif qui non seulement stimulent, mais transforment les formes de l'urbanisation. Ces réformes libéralisent partiellement le régime foncier, encouragent la promotion et les investissements immobiliers (dont les investissements étrangers). Durant la même période, de nouveaux schémas directeurs sont adoptés qui 
encouragent l'expansion spatiale des villes vietnamiennes. Les politiques d'urbanisme sont progressivement révisées au profit de l'activité immobilière, notamment afin de faciliter l'investissement et la construction de grands ensembles en zone périurbaine.

\section{Le recours à un nouveau modèle de production urbaine : les Nouvelles Zones Urbaines}

C'est dans la foulée de ces réformes que l'État-parti vietnamien adopte, au début des années 1990, un nouveau modèle de production urbaine appelé Nouvelle Zone Urbaine (NZU) ( $k h u$ do thi moi en vietnamien). Ce modèle prévoit la réalisation de grands ensembles périurbains principalement planifiés par les pouvoirs publics et vise à produire des zones multifonctionnelles à dominantes résidentielles. Le modèle des NZU émerge dans la foulée de l'abandon du précepte de production étatique des logements urbains, une politique que l'État n'arrivait plus à financer. À la différence des logements produits durant la période d'économie administrée, les logements construits dans les NZU ne sont plus réservés aux fonctionnaires ou employés d'État, ni assortis d'un faible loyer symbolique, mais vendu sur le marché. Ce modèle traduit également la volonté de l'État-parti de reprendre en main le développement urbain face au phénomène d'expansion urbaine spontanée qui prend forme au sortir des réformes à Hanoi comme à Ho Chi Minh Ville (Ha et Wong, 1999 ; Pandolfi, 2001).

Contrastant avec les pratiques d'auto-construction qui caractérisent alors la croissance des villes vietnamiennes, le modèle des NZU favorise la construction de grands ensembles sur des surfaces comprises entre 20 à 200 hectares, et parfois plus selon les orientations politiques validées en haut lieu. Les schémas directeurs des grandes villes vietnamiennes intègrent ce nouveau modèle dès la fin des années 1990, prescrivant l'aménagement de dizaines de ces NZU sur leurs marges. Au départ, ces ensembles sont planifiés, financés, construits et commercialisés par des sociétés étatiques (et leurs homologues actionnarisées ${ }^{3}$ ). Mais à Ho Chi Minh Ville des promoteurs privés entretenant des liens étroits avec les élites politiques commencent à produire des NZU au tournant des années 2000 (Kim, 2008 :132-140; Musil et al., 2019).

\section{Le foncier au service du financement de l'urbanisation}

11 En complément de l'adoption du modèle des NZU, l'État-parti engage une libéralisation contrôlée du foncier. Avant les réformes du Đổi Mới le foncier dans la ville socialiste n'avait pas de valeur (Kim, 2009). Son usage était alloué par acte administratif. Alors que la propriété privée du sol n'existe pas au Vietnam - la terre appartient au Peuple et l'État en assure la gestion, l'Assemblée nationale adopte en 1993 une loi foncière reconnaissant des Droits d'Usage du Sol (DUS). Les usagers des terrains possèdent ainsi des droits d'une durée déterminée ${ }^{4}$ et renouvelable. Cette loi concède indirectement une valeur au foncier (Pandolfi, 2001) faisant entrer le sol dans la sphère marchande ${ }^{5}$. Une première étape de la monétisation du foncier est franchie.

\section{Une fiscalité locale en cours d'adaptation}

12 Une seconde étape se produit en deux temps. D'abord, une nouvelle loi foncière adoptée en 2003 permet aux provinces de récupérer des droits d'usages de terrains (jusqu'à 200 hectares) et de les réaffecter à des promoteurs pour la réalisation de 
projets immobiliers (dont les NZU) (Labbé et Musil, 2014). Dans un deuxième temps, un amendement sur la loi budgétaire en 2004 donne aux provinces le droit de percevoir pour leur usage propre plusieurs taxes et redevances foncières ${ }^{6}$. Il s'agit de revenus substantiels. Entre 2008 et 2011, ils ont représenté $23 \%$ des revenus de Ho Chi Minh Ville (World Bank, 2013, p. 193). Ces rentrées fiscales restent néanmoins insuffisantes pour réaliser les infrastructures prévues par le schéma directeur actuellement en vigueur à Ho Chi Minh Ville.

\section{Le recours au dispositif terres-contre-infrastructure}

13 C'est face à ce manque à gagner qu'Ho Chi Minh Ville commence à utiliser un mécanisme de type land-based financing appelé au Vietnam terres-contreinfrastructures. Ce dispositif permet aux autorités (municipales ou étatiques) de réaffecter les droits d'usages de terrains constructibles à un promoteur (privé ou public, local ou étranger) en échange de la réalisation d'une infrastructure (route, pont, usine de traitement des eaux, etc.) ou d'un équipement public (école, parc, etc.). Ce dispositif est souvent utilisé pour la construction de routes à travers le montage de partenariats affichés au Vietnam comme public-privé bien que la partie privée soit souvent une entreprise d'État actionnarisée ou non (Musil et Perset, 2015). Cet outil permet aux villes de construire les infrastructures et les équipements prévus par les schémas directeurs tout en limitant leur contribution en capital et leur endettement (Labbé et Musil, 2014). En 2017, la municipalité de Ho Chi Minh Ville a émis une liste de 95 projets d'investissements dont la mise en œuvre prévoit le recours au dispositif décrit ci-dessus, pour un montant dépassant les 9 milliards de dollars US.

La mise en œuvre de ce dispositif de financement présente toutefois de nombreuses zones d'ombres et des incertitudes. Bien que la règlementation encourage la mise aux enchères des terrains prévus dans les contrats, l'attribution des projets se fait le plus souvent de gré à gré (Musil et Perset, 2015) ouvrant la porte à la corruption. Ensuite, les terrains proposés par la municipalité ne doivent pas être occupés. S'ils le sont, il revient (en théorie) aux pouvoirs publics d'engager l'acquisition des droits d'usage des terrains et de compenser leurs détenteurs. Les procédures de récupération des terres, en d'autres termes les procédures d'expropriation, sont généralement longues et très couteuses (notamment dû à l'augmentation des valeurs foncières). Enfin, l'évaluation des prix des terrains prévus dans les contrats pose problème. Un manque de transparence et une tendance à la sous-évaluation des valeurs foncières, favorisant les investisseurs au détriment des municipalités, ont été rapportés (World Bank, 2013 ; Nguyen et al., 2018).

15 C'est sur fond de transformation des politiques de développement urbain impliquant l'adoption de nouveaux modèles de production urbaine et d'outils de financement de l'urbanisation reposant sur la participation de nouveaux acteurs économiques (dont ceux des secteurs privé et étatique actionnarisé) que l'activité immobilière est devenue un enjeu majeur pour le développement de Ho Chi Minh Ville. Les pouvoirs publics ont eu besoin de s'appuyer sur ces acteurs afin d'assurer la production de la ville prévue par les plans d'aménagement. 


\section{Le district 2 et l'émergence d'un marché immobilier neuf}

16 Comme ailleurs en Asie, le "virage immobilier » vietnamien repose sur l'implication d'acteurs économiques $\mathrm{du}$ secteur de la promotion immobilière œuvrant principalement sur le segment résidentiel. Le district 2 de Ho Chi Minh Ville constitue une illustration de ce phénomène au regard du nombre d'opérations lancées, du nombre de logements livrés comme des formes urbaines produites tendant vers la verticalisation et la densification. Le segment résidentiel est en outre animé par une diversité d'acteurs, aussi bien locaux qu'étrangers.

\section{L'amorce de l'aménagement du district 2 et de la Nouvelle Zone Urbaine de Thủ Thiêm}

17 Le premier schéma directeur de la période post-Đổi Mới de Ho Chi Minh Ville est approuvé en 1993. Déjà à cette époque, les autorités visent à faire de la métropole du sud un pôle attractif pour les investisseurs étrangers dans le but de stimuler le décollage économique $d u$ pays. La planification du district 2, un secteur stratégiquement situé face au cœur historique de Ho Chi Minh Ville sur la rive opposée de la rivière Saigon, s'inscrit dans cette orientation. Le projet d'aménager la Nouvelle Zone Urbaine de Thủ Thiêm est approuvé en 1996. D'une surface de 737 hectares, cette NZU est vouée à devenir un futur centre financier, commercial et de services haut de gamme qui est censé pallier les lacunes fonctionnelles du cœur historique de la ville (Legoux, 2011). Cet aménagement est présenté par le gouvernement vietnamien comme «le Pudong de Ho Chi Minh Ville » et se veut la vitrine de la politique de modernisation de la métropole du sud (Quertamp, 2017).

Plusieurs mesures sont prises afin de réaliser ce projet porte-étendard, notamment la création d'un dispositif de pilotage du projet plus autonome et décentralisé. Placé en 2001 sous l'autorité de la municipalité de Ho Chi Minh Ville, le Thu Thiem Investment and Construction Authority (ICA) reçoit pour mandat de gérer l'aménagement dans le périmètre de la NZU et d'être une interface pour les investisseurs potentiels (Harms, 2016, p. 177). Malgré cette innovation institutionnelle, le démarrage de l'opération d'aménagement est flottant (Legoux, 2011). En cause, le lancement par la municipalité de plusieurs projets d'aménagement dans d'autres districts de la métropole créant de fait une situation de mise en concurrence.

Dès la fin des années 1990, Ho Chi Minh Ville approuve un deuxième grand projet d'aménagement dans un autre secteur de sa périphérie. Financée par un investisseur taiwanais (CT\&D Group), la NZU Saigon South prévoit l'aménagement de 3000 hectares au sud de l'agglomération (voir carte 1). En 2000, les pouvoirs publics lancent un concours international pour l'élaboration d'un plan de réaménagement des districts centraux de Ho Chi Minh Ville. Ces projets concurrencent directement le projet de Thủ Thiêm et l'aménagement $\mathrm{du}$ district 2 , drainant au contraire l'investissement immobilier vers le centre et le sud de la ville (Huynh, 2015b; Harms, 2016 ; Quertamp, 2017). En outre, en raison de difficultés d'accès au foncier dans le secteur de Thủ Thiêm, les investisseurs se sont davantage intéressés aux projets situés au sud de la métropole. L'aménagement de la NZU de Thủ Thiêm et la construction des infrastructures de liaison (ponts et tunnel) devant relier le district 2 au centre-ville impliquent en effet 
des d'expropriations. Les plans d'aménagement font par ailleurs fi des quelques 14000 ménages qui vivent dans les quartiers spontanés qui ont pris forme sur le territoire (Harms, 2016, p. 10). Engagées dès 2002, les procédures d'expropriation se prolongent jusqu'en 2014 et leur déroulé reste sujet à controverses (Vasavakul, 2019).

Dans le district 2, les opérations immobilières des grands promoteurs (privés ou anciennement liés à l'État) ne décolleront véritablement qu'après le lancement de projets d'infrastructures structurants financés par la municipalité et l'aide publique japonaise, dont la première ligne de métro et la construction d'une autoroute en prévision de la réalisation du nouvel aéroport situé à l'est de la métropole.

\section{Une montée en intensité rapide de l'activité immobilière...}

21 À l'exception d'un grand ensemble (la NZU Phu Anh Khanh), le district 2 n'affiche, jusqu'au milieu des années 2000, qu'une dizaine d'opérations immobilières commerciales d'envergure modeste. La dynamique commence à changer à la fin des années 2000, période qui voit l'engagement des premières opérations sous forme de joint-venture associant des promoteurs étrangers. Le lancement de ces opérations coïncide avec l'adhésion du Vietnam à l'Organisation mondiale du commerce, adhésion qui aura pour effet de gonfler les investissements directs étrangers dans le secteur immobilier de Ho Chi Minh Ville (Le Thanh Tung, 2016).

Jusqu'en 2010 seules 23 opérations commerciales avaient été lancées et réalisées, couvrant au total environ 50 hectares seulement à l'échelle du district qui en compte 5 000. Entre 2011 et 2013, bien que le marché immobilier à l'échelle du pays connût un ralentissement important, subissant indirectement l'onde de choc de la crise des subprimes (Do et al., 2015), plusieurs opérations ont été lancées. Parmi la quinzaine de projets engagés apparaissent les premiers projets d'envergure pour la NZU de Thủ Thiêm. Officiellement approuvé en 2012, le projet Sala, du promoteur Đại Quang Minh, constitue l'opération levier qui confirme le début de l'aménagement tant attendu de Thủ Thiêm. Malgré leur nombre limité, les opérations engagées durant cette période mobilisent plus de 180 hectares à l'échelle du district.

Le marché immobilier connaît un rebond à partir de 2014 (HoREA, 2019), entraînant une augmentation rapide du nombre d'opérations approuvées par les autorités : 53 chantiers sont lancés, dont 31 sont toujours en cours (en 2019) - ces opérations se réalisant par phase, en fonction des capitaux dont disposent les promoteurs et des opportunités qu'offre le marché. Entre 2014 et 2019, ce sont près de 310 hectares de terrains qui ont été valorisés par la construction d'opérations résidentielles. En 2019, une quinzaine d'opérations étaient planifiées et en attente de démarrage, celles-ci couvrant plus de 400 hectares sur l'ensemble du district. 


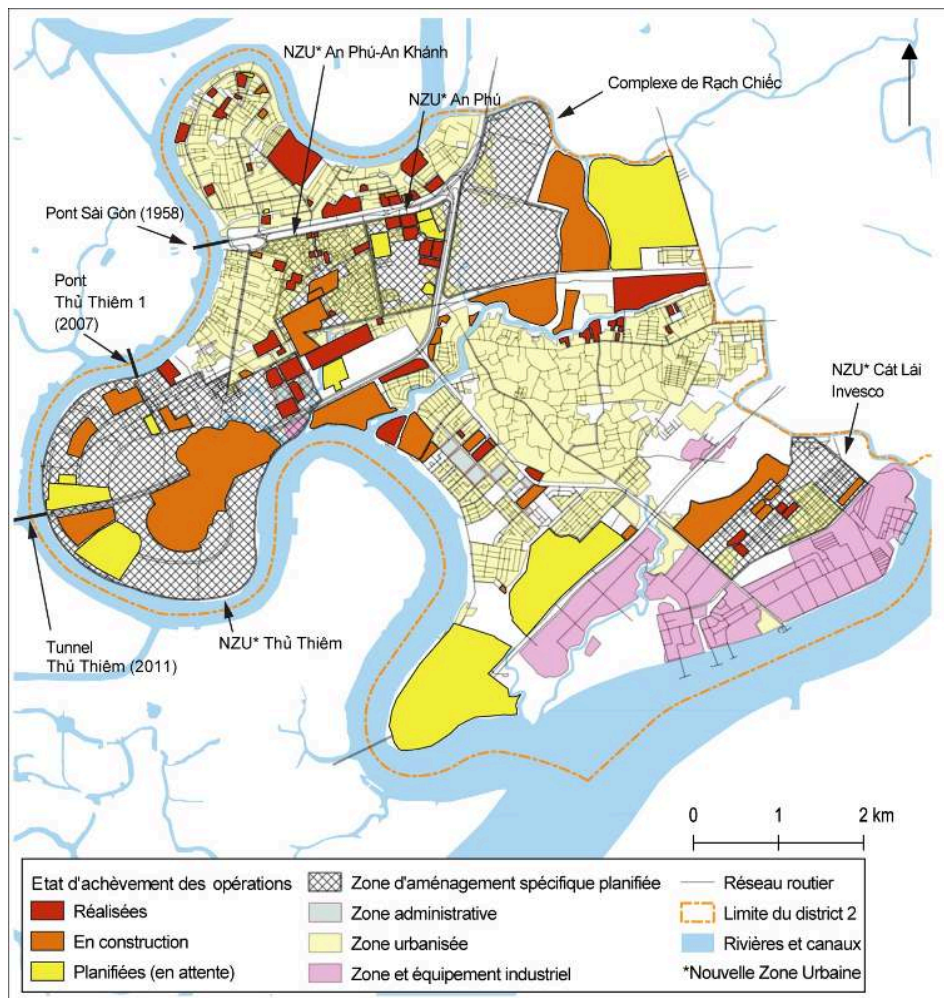

Auteurs : Musil, Labbé et Vu, 2020. NZU de Thủ Thiêm, les documents de planification approuvés à l'échelle du district 2 prévoient le développement de nouveaux quartiers résidentiels. Cela passe par la construction d'ensembles de logements impliquant la conversion d'emprises agricoles et par des projets de renouvellement urbain - ce qui signifie faire table rase d'un tissu urbain existant jugé dégradé. Les nouveaux projets immobiliers ne prennent donc pas spécifiquement place dans des zones d'aménagement planifiées comme les NZU, mais sur l'ensemble du territoire du district.

En 2019, on recense à l'échelle du district une douzaine d'opérations immobilières affichant des surfaces de 20 hectares et plus (voir illustration 4). Au regard des surfaces affichées, ces opérations pourraient entrer dans la catégorie des NZU (telle que définie en section 1). Hormis la construction de logements de type maison individuelle («villa »), maison mitoyenne et tour d'appartements, ces opérations s'accompagnent d'équipement et de services (parcs, équipements sportifs, commerces, etc.) et de la réalisation des réseaux techniques (Voirie, Réseau Divers - VRD). Le district comprend une autre douzaine d'opérations, qualifiées ici de "moyennes », c'est-à-dire affichant les attributs d'une NZU, mais présentant une surface inférieure au seuil de 20 hectares. Enfin, plus de 80 opérations qualifiées ici de "petite échelle ", c'est-à-dire de moins de 5 hectares, ont été recensées. Ces opérations prennent, dans la majorité des cas, la forme de tours d'habitation présentant de fortes densités (illustrations 5, 6 et 7). À l'échelle du district, ces opérations ne couvrent pas plus de 130 hectares.

Or ce type de développement s'est traduit en vingt ans par la production d'environ 50000 logements, sous forme de tours d'habitation dont certaines de 50 étages ; ces opérations présentent de fortes densités pouvant atteindre 3500 habitants à l'hectare.

EchoGéo, 52 | 2020 
La construction de la ligne de métro traversant le district 2 a conduit la municipalité à accorder des dépassements de coefficient d'occupation des sols, donc la construction de tours de grande hauteur, pour les opérations à proximité des futures stations. La densification de ces secteurs est justifiée par un accès facilité aux futurs habitants à un transport public de masse. (Figure 3 et carte 4 ). Si les quartiers à haute densité sont déjà érigés, l'achèvement de la ligne de métro se fait attendre.

Illustration 4 - Classification des opérations immobilières engagées depuis 1999

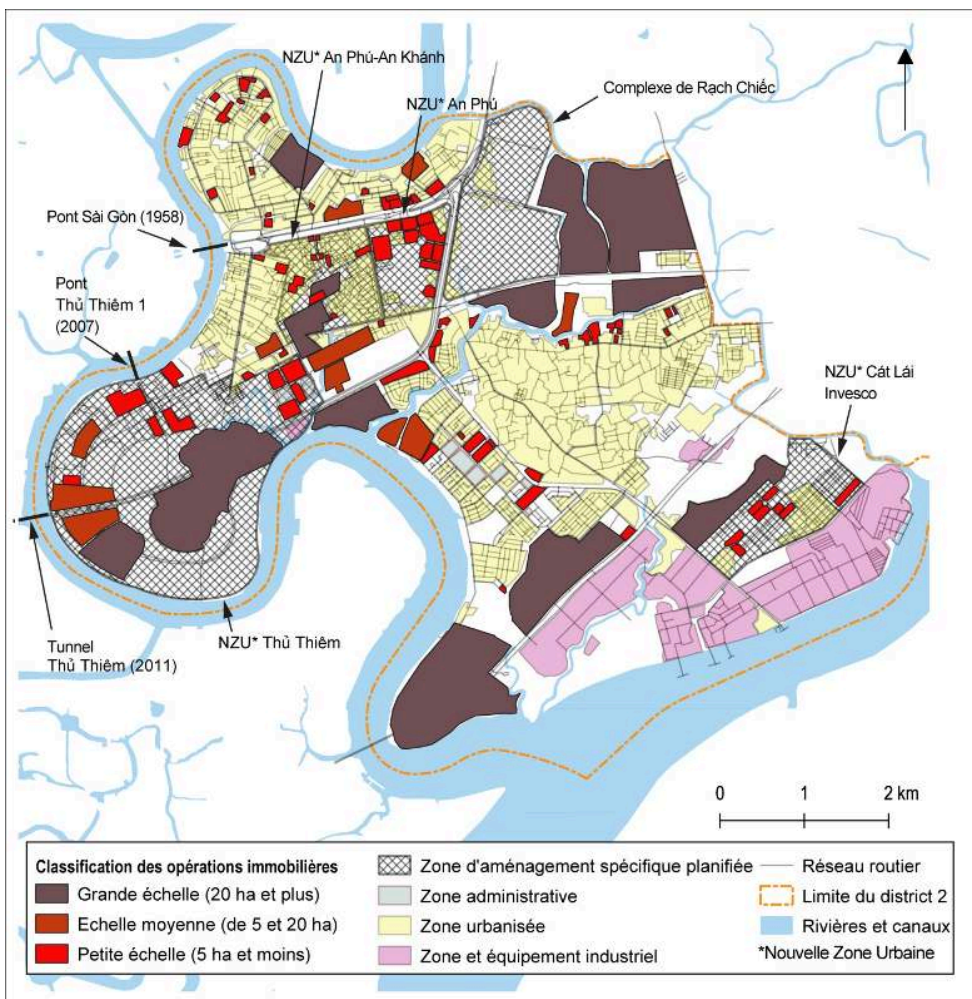

$\mathrm{Vu}, 2020$ 
Illustration 5 - Population potentielle après réalisation des opérations immobilières engagées depuis 1999

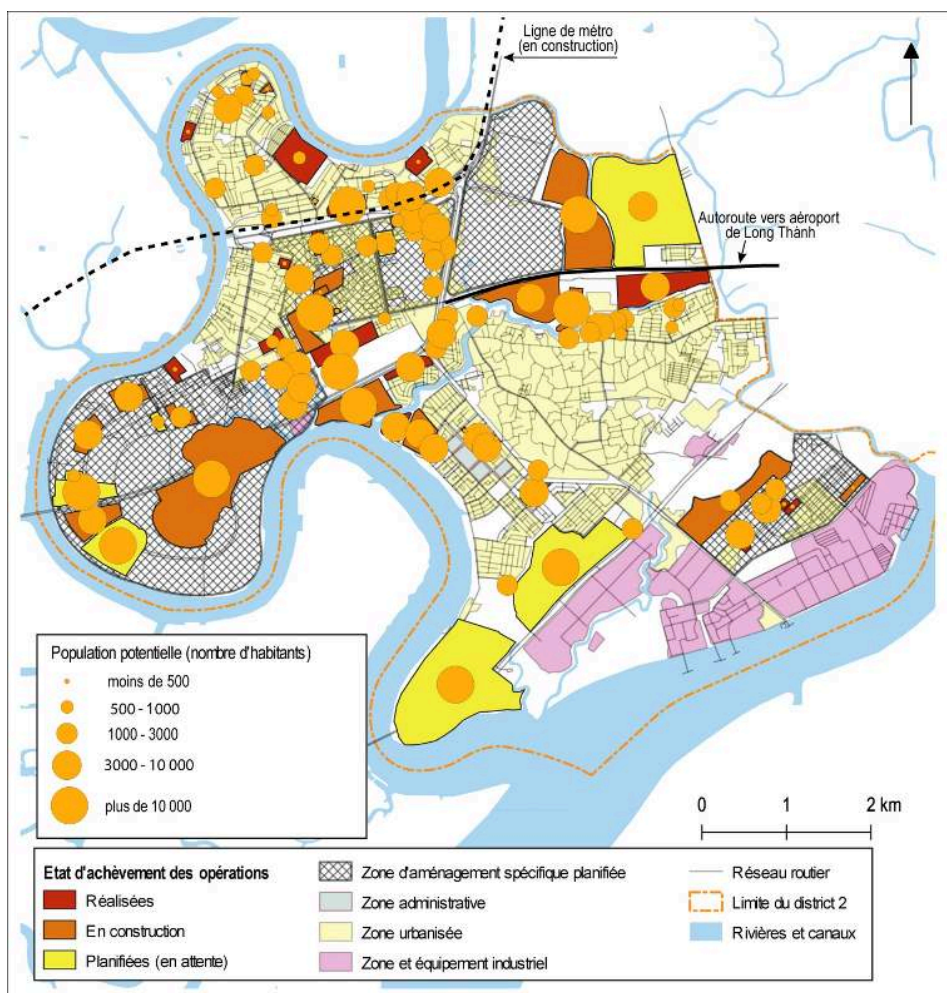

Auteurs : Musil, Labbé et Vu, 2020)

Illustration 6 - Nouveaux quartiers résidentiels présentant des densités d'environ 3500 habitants à I'hectare

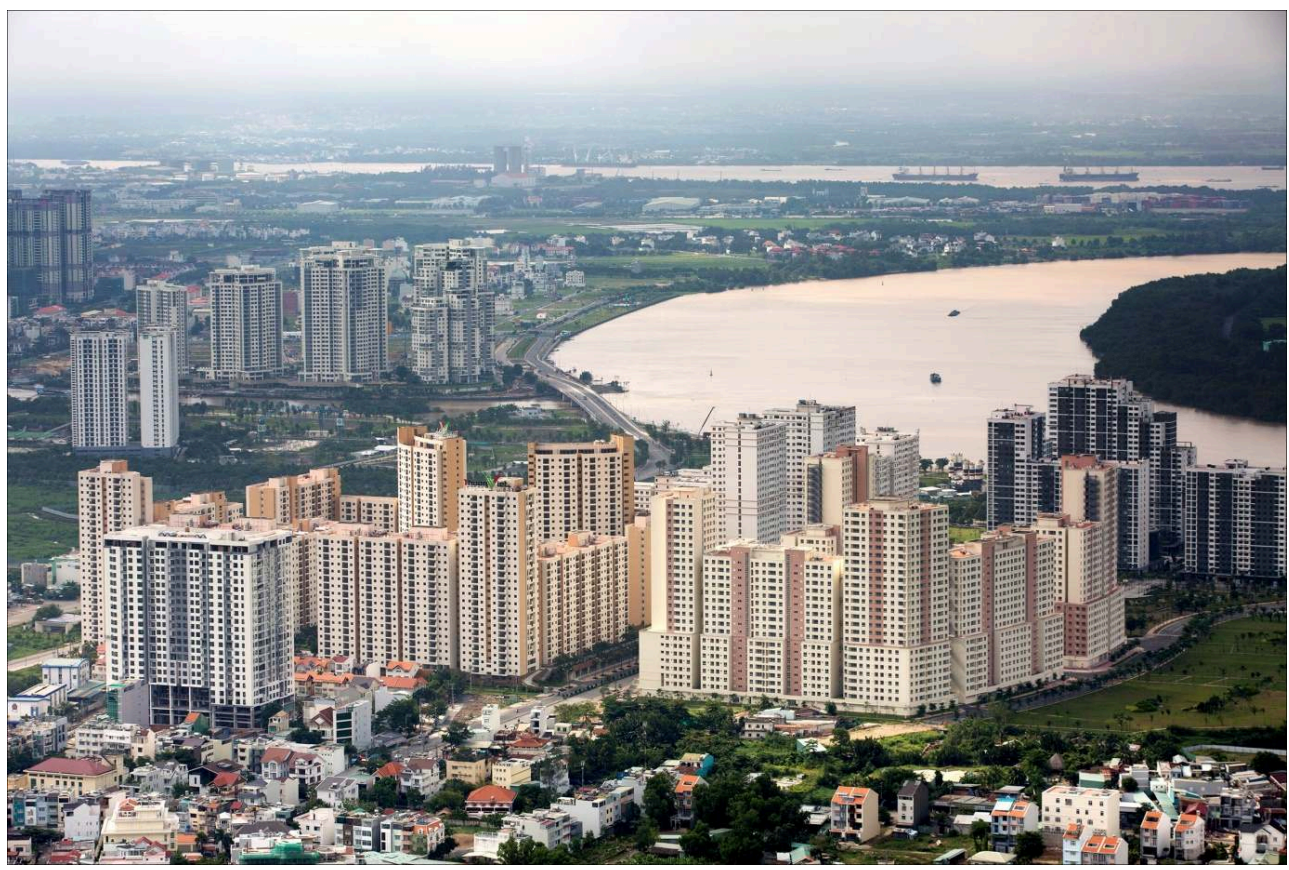

Auteur : Musil, 2018 


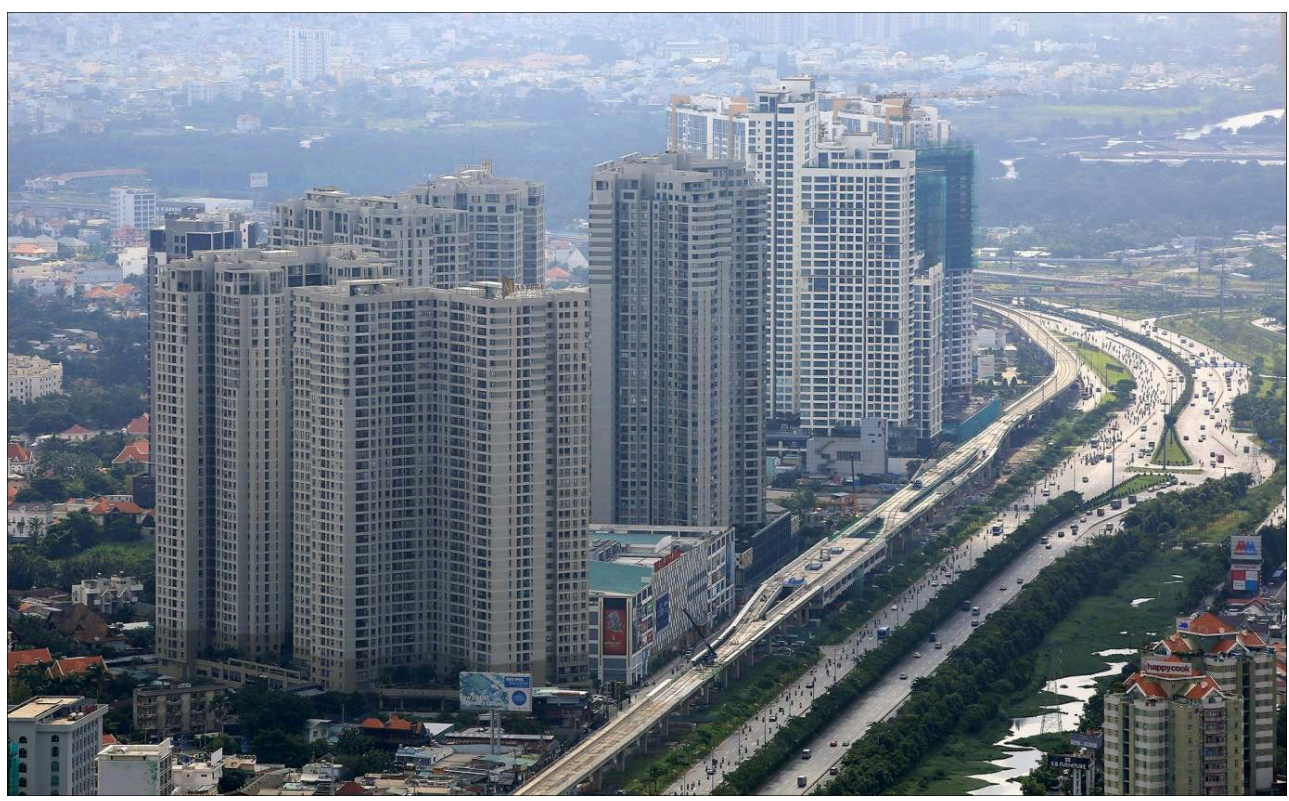

Auteur : Musil, 2018.

\section{...animée par une diversité de promoteurs et marquée par un décrochage entre l'offre et la demande}

La majorité des opérations commerciales du district 2 (au total 105) ont été ou sont présentement conduites par des acteurs relevant du secteur privé (voir tableau 1). Hormis quelques opérations, les sociétés étatiques sont très peu présentes. Pour autant, cela ne signifie pas que ces acteurs soient absents du marché. Bien au contraire. L'analyse de plusieurs opérations confirme la présence, en arrière-plan, d'anciennes sociétés étatiques aujourd'hui actionnarisées. S'ils sont officiellement considérés comme appartenant au secteur privé, ces acteurs ont conservé des liens étroits avec les organisations publiques (société étatique «mère » ou ancienne institution de tutelle), ce qui facilite leur accès au foncier (Kim, 2008, p. 34-51). Les sociétés étatiques actionnarisées sont particulièrement efficaces pour acquérir des actifs fonciers appartenant à des entités publiques, réaliser des opérations d'assemblage de parcelles ou encore obtenir des terrains publics directement auprès des autorités. D'autres acteurs privés (de "type 1 ", cf. tableau 1 ) excellent également dans ce domaine et constituent des réserves foncières. Un promoteur, Novaland, possède déjà 6 opérations dans le district 2 et dispose en plus d'environ 230 hectares (Novaland, 2019) à construire ou à revendre. 
Tableau 1 - Typologie des promoteurs impliqués dans des opérations immobilières commerciales dans le district 2

\begin{tabular}{|c|c|c|c|}
\hline $\begin{array}{c}\text { Type } \\
\text { d'entité }\end{array}$ & Description & $\begin{array}{l}\text { Nb. estimé } \\
\text { de projets }\end{array}$ & Commentaires \\
\hline $\begin{array}{l}\text { Société } \\
\text { étatique }\end{array}$ & $\begin{array}{l}\text { Entreprise à capitaux publics } \\
\text { établis organisée, gérée et } \\
\text { enregistrée pour fonctionner } \\
\text { conformément aux directives de la } \\
\text { planification étatique. L'entreprise à } \\
\text { l'origine provient souvent du } \\
\text { secteur de la construction. }\end{array}$ & 4 & $\begin{array}{l}\text { Ces promoteurs sont liés soit au Comité } \\
\text { Populaire de Ho Chi Minh Ville, à des } \\
\text { organisations publiques (comme le } \\
\text { "Front de la Patrie ») ou à des ministères } \\
\text { (comme le Ministère de la Construction). }\end{array}$ \\
\hline \multirow[b]{2}{*}{$\begin{array}{l}\text { Société } \\
\text { privée }\end{array}$} & $\begin{array}{l}\text { Type 1: entreprise à capitaux } \\
\text { privés spécialisée dans la } \\
\text { promotion immobilière. }\end{array}$ & \multirow[t]{2}{*}{73} & \multirow{2}{*}{$\begin{array}{l}\text { Ce sont les promoteurs les plus présents } \\
\text { dans le district } 2 \text {. } \\
\text { Toutefois la distinction entre promoteurs } \\
\text { privés et promoteurs issus d'entreprises } \\
\text { actionnarisées est difficile. } \\
\text { Bien souvent des sociétés privées de } \\
\text { projet impliquant un ou plusieurs } \\
\text { investisseurs/promoteur sont créées pour } \\
\text { une opération unique. L'identification des } \\
\text { acteurs est rendue compliquée en raison } \\
\text { des multiples entités associées à ces } \\
\text { montages et des participations croisées. }\end{array}$} \\
\hline & $\begin{array}{l}\text { Type 2: entreprise dite } \\
\text { «actionnarisée " constitue une } \\
\text { société par actions dont une partie } \\
\text { (ou la totalité) des actionnaires sont } \\
\text { des sociétés étatiques ou des } \\
\text { organisations autorisées par l'État. } \\
\text { Leur fonctionnement est similaire à } \\
\text { une entreprise privée à capitaux } \\
\text { privés. }\end{array}$ & & \\
\hline $\begin{array}{l}\text { Joint } \\
\text { Venture } \\
\text { étrangère }\end{array}$ & $\begin{array}{l}\text { Entreprise étrangère qui s'associe } \\
\text { avec un ou des investisseurs } \\
\text { vietnamiens pour développer des } \\
\text { projets résidentiels, entre autres } \\
\text { activités d'investissement. }\end{array}$ & 28 & $\begin{array}{l}\text { Les promoteurs actifs proviennent de } \\
\text { Singapour (Keppel, Capitaland) de Hong } \\
\text { Kong (Hong Kong Land, Chiap Hua, } \\
\text { Hamon Investment) et de Corée du Sud } \\
\text { (GS, Lotte, Daewon). }\end{array}$ \\
\hline
\end{tabular}

Auteurs : Musil, Labbé et Vu, 2020. la plupart des liens privilégiés avec des décideurs politiques et des fonctionnaires de haut rang (Kim, 2008). La plupart d'entre-elles engagent des partenariats avec d'autres acteurs du secteur immobilier présentant un savoir-faire reconnu en matière de construction, de suivi de projet ou de commercialisation. Ces partenariats prennent la forme de sociétés-projets aux participations croisées, dans le cas d'acteurs locaux, ou encore de joint-venture dans le cas de partenaires étrangers. Dans les montages impliquant un partenaire étranger, le partenaire local contribue au capital de la jointventure par l'apport du foncier. Le partenaire étranger apporte quant à lui des financements, une expertise et un argument de vente: une réputation internationale souvent considérée par les acheteurs comme un gage de qualité. Au total 28 projets joint-venture ont été recensés dans le district 2.

Le montage d'opérations de type joint-venture est encouragé par le gouvernement vietnamien qui cherche à attirer les investissements étrangers dans le secteur immobilier et parait, au moins dans la forme, imposer moins d'obstacles à la participation étrangère dans ce secteur par rapport aux autres marchés en Asie du SudEst (PWC, 2019). En retour, la constitution de joint-venture est recherchée par les investisseurs étrangers, plutôt que l'investissement via des sociétés 100 \% étrangères, davantage exposées à la complexité bureaucratique. La majorité des investisseurs étrangers présents sur le marché de Ho Chi Minh Ville sont des acteurs régionaux (Singapour, Hong Kong, Taiwan ou Corée du Sud) initiés aux pratiques locales; ils sont présents depuis l'ouverture économique du pays et partagent une culture des affaires commune avec leurs homologues vietnamiens (Kim, 2008).

marché immobilier de la métropole du sud est attractif pour ces promoteurs étrangers. Dans l'aire Asie Pacifique, Ho Chi Minh Ville apparait en 2019 au $7^{\text {ème }}$ rang des métropoles présentant les plus grandes opportunités d'investissement et au 
$2^{\text {ème }}$ rang des métropoles affichant un fort potentiel de développement (PWC, 2019). Avec une population de 10 millions d'habitants (en constante augmentation), la population de Ho Chi Minh Ville se caractérise par sa jeunesse (la moitié a moins de 35 ans) et par ses revenus deux à trois fois supérieurs à la moyenne nationale ${ }^{7}$. Pour les promoteurs, locaux comme étrangers, Ho Chi Minh Ville constitue un marché porteur en raison de la hausse du nombre de ménages en recherche de logement et en capacité d'investir. Encore faut-il que l'offre rencontre la demande.

Le marché immobilier résidentiel au Vietnam d'une manière générale et celui de Ho Chi Minh Ville en particulier présente en ce sens un fort décrochage (Pham Thai Son, 2014). La production de logements neufs s'oriente vers les segments milieu et haut de gamme, affichant des prix d'entrée équivalents à $800 \mathrm{USD} / \mathrm{m}^{2}$, alors que la production d'opérations dites abordables, avec des prix d'entrée au $\mathrm{m}^{2}$ inférieurs à 800 USD, est limitée (voir tableau 2). Or les besoins des ménages urbains de la métropole correspondent davantage au segment abordable. Ce qui laisse apparaitre un écart préoccupant entre les besoins, non satisfaits, pour une majorité de la population et une offre en logement neuf inadaptée (Pham Thai Son ; 2014 ; HoREA, 2019).

Tableau 2 - Logements commerciaux neufs mis en vente entre 2015 et 2018 à Ho Chi Minh Ville (par segment de marché)

\begin{tabular}{|l|c|c|c|c|c|c|c|c|}
\hline & \multicolumn{2}{|c|}{2015} & \multicolumn{2}{c|}{2016} & \multicolumn{2}{c|}{2017} & \multicolumn{2}{c|}{2018} \\
\hline $\begin{array}{c}\text { Nombre d'unités produites } \\
\text { mis en vente }\end{array}$ & \multicolumn{2}{|c|}{40000} & \multicolumn{2}{c|}{38000} & \multicolumn{2}{c|}{31000} & \multicolumn{2}{c|}{31000} \\
\hline $\begin{array}{l}\text { Segment luxe (prix supérieur à } 80 \\
\text { millions VND/m² } 3500 \text { USD/m²) }\end{array}$ & 400 & $1 \%$ & 1520 & $4 \%$ & 310 & $1 \%$ & 930 & $3 \%$ \\
\hline $\begin{array}{l}\text { Segment haut de gamme (prix } \\
\text { de } 34 \text { à } 80 \text { millions VND/m² entre } \\
1500 \text { et 3 500 USD/m²) }\end{array}$ & 15200 & $38 \%$ & 13300 & $35 \%$ & 6820 & $22 \%$ & 13300 & $43 \%$ \\
\hline $\begin{array}{l}\text { Segment milieu de gamme (prix } \\
\text { de } 18 \text { à 34 millions } / \mathrm{m}^{2}=\text { entre } 800 \text { et } \\
1500 \text { USD } / \mathrm{m}^{2} \text { ) }\end{array}$ & 16000 & $40 \%$ & 18620 & $49 \%$ & 19840 & $64 \%$ & 16120 & $52 \%$ \\
\hline $\begin{array}{l}\text { Segment abordable (prix } \\
\text { inférieur à } 18 \text { millions VND/m² } \\
\left.\text { USD } / \mathrm{m}^{2}\right)\end{array}$ & 8400 & $21 \%$ & 4560 & $12 \%$ & 4030 & $13 \%$ & 620 & $2 \%$ \\
\hline
\end{tabular}

Source : CBRE - Vietnam Real Estate Market Outlook 2019 - Ho Chi Minh City - 09/01/2019.

Auteurs: Musil, Labbé et Vu, 2020.

Le décrochage décrit plus haut à l'échelle de la métropole est exacerbé dans le district 2. D'abord, en 2019, le prix médian au $\mathrm{m}^{2}$ d'un appartement (tout segment confondu) était de 40 millions VND $/ \mathrm{m}^{2}$ (soit $1700 \mathrm{USD} / \mathrm{m}^{2}$ ). Cela indique que la plupart des projets achevés, en cours de construction ou en attente de démarrage, relèvent des catégories supérieures. En outre, les prix des unités haut de gamme neuves ont connu une hausse de $52 \%$ depuis 2016 (DKRA, 2019). Les nouveaux projets en attente de lancement, notamment ceux présentant des localisations de choix, spécialement dans la NZU de Thủ Thiêm, affichent des prix de vente allant de 55 à plus de 85 millions $\mathrm{VND} / \mathrm{m}^{2}$ (soit entre 2400 et $4000 \mathrm{USD} / \mathrm{m}^{2}$ ) pour des appartements. Le prix des maisons individuelles ( villa )) et maisons mitoyennes atteignent des valeurs comprises entre 130 et 250 millions $\mathrm{VND} / \mathrm{m}^{2}$ (soit 5600 et $10800 \mathrm{USD} / \mathrm{m}^{2}$ ). Précisions que l'écart entre les prix au $\mathrm{m}^{2}$ des villas et maisons mitoyennes d'un côté et des appartements de l'autre s'explique par le cout élevé du foncier. A l'opposé des opérations milieu et haut de gamme apparaissent les opérations de logements dits abordables. Une douzaine 
d'opérations réalisées au cours de ces dix dernières années entrent dans ce segment, ce qui représente un peu moins de 6000 unités, avec des prix compris entre 21 et 28 millions $\mathrm{VND} / \mathrm{m}^{2}$ (soit entre 900 et $1200 \mathrm{USD} / \mathrm{m}^{2}$ ).

Le décrochage entre l'offre et la demande en logement s'explique par plusieurs raisons, nous en exposons ici trois. Tout d'abord, comme indiqué précédemment, le prix final des produits immobiliers dépend du prix du foncier8. Les coûts de construction sont assez faibles au Vietnam - de l'ordre de $600 \mathrm{USD} / \mathrm{m}^{2}$. Le foncier ayant une valeur plus élevée que les constructions, ce coût affecte le prix final d'un bien. Dans les modèles économiques des promoteurs, le coût du foncier varie entre 30 et $50 \%$ du coût total de l'opération; les coûts de construction, incluant l'aménagement des VRD, représentent 50 à $70 \%$. Les autres coûts de transaction, notamment ce qui est qualifié de lobby money (montants distribués à des intermédiaires dans le but d'accélérer le traitement administratif des projets) sont difficilement quantifiable (et non divulgués), mais sont toutefois pris en compte dans l'équilibre budgétaire des opérations. La plupart des promoteurs engageant des projets commerciaux visent une rentabilité de l'ordre de $20 \%$, avec un retour sur investissement rapide, après la vente sur plan des logements. Le coût du foncier ainsi que les pratiques des promoteurs expliquent (en partie) la formation des prix de vente finaux.

La seconde raison expliquant un décrochage entre l'offre et la demande en logement concerne les acheteurs. Les promoteurs identifiés comme les plus agressifs sur le marché ciblent deux types d'acheteurs capables d'investir dans les segments milieu et haut de gamme. On trouve d'abord les acheteurs "spéculateurs». Il s'agit de particuliers, seuls ou en groupe, qui achètent sur plan une ou plusieurs unités et les revendent au fur et à mesure de l'avancée de la construction de l'opération. Les biens peuvent également être conservés, en vue notamment de la montée des prix attendue dans le district 2 avec la réalisation de la ligne de métro. Ces biens peuvent également être conservés comme des placements et relèvent alors d'une stratégie d'investissement familial. Enfin, ils peuvent être mis en location, à destination d'étrangers ou en location saisonnière (type AirBnB). Certains courtiers en immobilier rapportent que la grande majorité de leurs clients (donc des acheteurs) appartiennent à la catégorie des spéculateurs (entre 50 et $75 \%$ des acheteurs).

Parmi les acheteurs des segments supérieurs, on retrouve les utilisateurs finaux. C'està-dire les acheteurs souhaitant acquérir un logement pour l'occuper. Pour ce type d'acheteur, les promoteurs ne s'appuient pas forcément sur leur capacité d'achat actuel, mais plutôt sur leur effort financier potentiel. Certains promoteurs visent une montée en gamme et une montée des prix sachant que la population est jeune (et active), qu'elle s'enrichit, qu'elle souhaite consommer des produits de meilleure qualité et qu'elle dispose de capacités d'emprunt auprès des banques. Ce qui est vrai pour les biens de consommation usuels s'applique à l'immobilier. Nombreux sont les promoteurs tenant ce discours les poussant ainsi à développer des opérations à forte rentabilité.

La troisième raison expliquant les prix élevés des logements neufs mis en vente dans le district 2 renvoie à des décisions politiques. Depuis 2017, la production de logements neufs ralentie à Ho Chi Minh Ville (cf. tableau 2). Cela fait suite à l'adoption en 2017 d'une décision de la municipalité de suspendre plus de 150 opérations, celles-ci présentaient des irrégularités liées aux procédures d'investissement. Plus précisément une série d'inspections a été engagé afin de s'assurer de la régularité des droits d'usage 
des terrains, notamment pour les terrains précédemment considérés comme " public » (terrains appartenant à la municipalité ou à un district) ou comme "actif public » (terrains appartenant à des sociétés étatiques). En fait ces inspections constituent la mise en application des directives anticorruptions impulsées par le Premier Ministre et visant à assainir le marché immobilier (Le Hong Hiep, 2019). Or cette décision a eu des répercussions notables à Ho Chi Minh Ville. La municipalité ayant adopté des mesures complémentaires suspendant la réalisation d'opérations dans les districts centraux et imposants la révision des procédures administratives des opérations suspectes à l'échelle de la métropole. Depuis 2019, on observe une diminution du nombre d'opérations approuvées par la ville, ralentissant la construction et la livraison des logements neufs. Cela s'est traduit par une hausse des prix immobiliers et par une baisse des revenus pour la municipalité - les revenus liés au foncier ayant diminués de 22,5\% depuis 2017 (HoREA, 2019).

\section{Les symptômes de l'intensification du développement immobilier et les conflits liés à l'aménagement de la NZU de Thủ Thiêm}

37 L'intense développement immobilier du district 2, l'envolée des prix des logements neufs et la multiplication du nombre d'opérations et de promoteurs constituent autant de marqueurs de la profonde transformation des politiques de développement urbain et des pratiques de planification au Vietnam. Cette intensification n'est pas dénuée de tensions ni de conflits. Depuis 2018, concomitamment à l'aménagement de la NZU de Thủ Thiêm et au développement immobilier dans le district 2, deux types de conflits émergent. Ils sont symptomatiques de l'intensification de l'activité immobilière et de ses dérives. Le premier est indirectement lié aux procédures d'expropriation et pointe les irrégularités en matière de planification. Le second concerne les contrats impliquant le dispositif terres-contre-infrastructure évoqué en section 1 et questionne les modes d'évaluation des prix du foncier entrant dans ce type d'échange.

\section{Une planification urbaine précise, mais des périmètres opérationnels flous}

Comme évoqué plus haut, l'engagement de l'aménagement de la NZU de Thủ Thiêm a suscité de vives protestations de la part des habitants devant être déplacés (Harms, 2016). Ces contestations concernaient principalement deux aspects : le montant des compensations (jugées inférieures à la valeur marchande des terrains récupérés par les autorités) et les modalités de réinstallation (dans des zones de relogement considérées comme trop éloignées du lieu de vie initial et provoquant la perte des sources de revenus pour de nombreux ménages) (Harms, 2016). ICA (Thu Thiem Investment and Construction Authority) qui pilotait ces procédures s'est heurté à une résistance tenace des habitants (Legoux, 2011) ce qui a conduit à un allongement des procédures jusqu'en 2014.

En 2018, un scandale lié à la planification de la NZU, et plus particulièrement à l'ajustement du périmètre de cette zone, a été porté à connaissance dans la presse. Dans sa forme initiale, approuvée en 1996, l'opération présentait une surface de 770 hectares 
(comprenant des zones lacustres non urbanisables) et une zone de relogement de 160 hectares pour les habitants déplacés. Par la suite, la municipalité a modifié le périmètre de la zone et les fonctions de terrains adjacents au projet central d'aménagement d'un nouveau Central Business District. En 2005, la municipalité a ajusté le plan revoyant à la baisse la surface de la zone (737 hectares, dont 657 de NZU et 80 hectares de zone urbaine à rénover) (Thanh Niên, 2018). La nouvelle décision s'imposant à la précédente. Or dans les faits, le périmètre d'expropriation a finalement été plus large que celui prévu en 2005 , tout en étant moins vaste que celui initialement établi en 1996. Au final, certains ménages ont été expropriés alors que le plan initial ne le prévoyait pas. Un plan d'aménagement élaboré en 1996 présentant le périmètre exact du projet de NZU est réclamé pour vérification par des habitants spoliés. Or les autorités n'ont pas été en mesure de présenter ce document - du moins son original signé de la main du Premier Ministre et faisant autorité (Thanh Niên, 2018).

La contestation qui s'organise a posteriori des procédures d'éviction porte sur les prix bas de compensations (en comparaison avec les prix de revente élevés des terrains dont les DUS ont été réalloués à des promoteurs) et surtout pointe la réquisition arbitraire de surfaces hors du périmètre de la NZU (Thanh Niên, 2018). Plusieurs pétitions ont été adressées à la municipalité et des actions en justice ont été intentées. Comme un blogueur le rapporte au sujet d'une habitante, sa maison située sur un axe existant de la zone d'aménagement, dont le terrain valait en 2018 près de 200 millions $\mathrm{VND} / \mathrm{m}^{2}$ $\left(\sim 8500 \mathrm{USD} / \mathrm{m}^{2}\right)$ a été compensée à hauteur de 18 millions $\mathrm{VND} / \mathrm{m}^{2}$ (soit $800 \mathrm{USD} / \mathrm{m}^{2}$ ) quelques années auparavant. Cette dernière a été prioritaire pour l'achat d'un d'appartement de réinstallation cédé à 20 millions $\mathrm{VND} / \mathrm{m}^{2}$ (soit $900 \mathrm{USD} / \mathrm{m}^{2}$ ). Comme cette habitante, de nombreux ménages ont perdu leur logement, souvent aussi le lieu de leurs activités économiques, endossé des dettes (en raison de l'achat d'un logement de réinstallation plus cher que le montant des compensations perçues), alors que leur bien exproprié était situé en dehors de la zone de planification initiale (Tiếng Dân, 2018).

41 En 2018, le Premier Ministre a chargé le gouvernement d'examiner les causes ayant suscité le lancement de pétitions. L'inspection a conclu qu'une superficie de 4,3 hectares située en dehors de la zone du projet avait été expropriée. D’après les explications officielles, cette zone supplémentaire avait été ajoutée au plan dans le cadre d'un autre ajustement réalisé en 1998 par l'architecte en chef de Ho Chi Minh Ville, alors que ce dernier n'avait pas compétence pour approuver cet ajustement. L'inspection conduite a également évoqué des erreurs dans les modalités de récupération de terrains, non conforme à la réglementation. Les anciens résidents ont rejeté ces conclusions, insistant sur le fait que la superficie des terres expropriées en dehors du périmètre prévu était supérieure aux 4,3 hectares identifiés (Thanh tra Chính phủ, 2018). En outre, entre 1996 et 2016, il n'y a pas eu d'ajustement officiellement validé par l'ensemble des instances décisionnaires de Ho Chi Minh Ville (Vasavakul, 2019).

42 Malgré les exercices de contrition de responsables politiques (Nhóm Phóng Viên, 2018), ce conflit, toujours non résolu, suggère à quel point l'ajustement des schémas d'aménagement s'opère dans l'opacité. En outre, ce cas montre qu'il n'existe aucun mécanisme de recours par rapport aux procédures d'expropriation et d'indemnisation. Les demandes par voie administrative ne constituent pas des recours efficaces pour traiter les griefs, car les organismes publics ont tendance à se protéger mutuellement 
aux dépens des intérêts des habitants (Vasavakul, 2019) affectés par des procédures jugées injustes (Tiếng Dân, 2018).

\section{Des échanges de terres-contre-infrastructure déséquilibrés} terres-contre-infrastructure. Le nœud du problème porte sur l'évaluation des prix du foncier introduit dans ces échanges. Après récupération des terrains dans le périmètre de la NZU de Thủ Thiêm par les pouvoirs publics, la municipalité a constitué un fonds foncier de 221 hectares. Ce fonds était destiné à financer les infrastructures dont l'investissement serait engagé sous la forme de projets terres-contre-infrastructure (Nhật Minh, 2019). La réalisation des principales infrastructures (ponts, routes, assainissement) de la NZU de Thủ Thiêm a été attribuée à deux sociétés.

première, Đai Quang Minh, une société privée, a reçu plus de 105 hectares de terrains à construire en l'échange de la réalisation de quatre routes (soit $12 \mathrm{~km}$ de voirie), d'un pont routier, de l'aménagement de l'espace public principal de la NZU et d'un autre parc le long de la rivière. Actuellement, ce foncier est en cours de valorisation avec la construction du projet immobilier Sala mentionné plus haut. La seconde, CII (Ho Chi Minh City Infrastructure Investment), a reçu environ 10 hectares pour la réalisation de voirie interne à de futurs îlots de la NZU (Tầm Nhìn, 2019). Précisons que si CII est une société privée, elle a néanmoins été créée par la municipalité en 2001 pour lever des capitaux sur les marchés financiers dans le but d'investir dans la construction d'infrastructures. CII prévoit de développer une partie des terrains ainsi acquis à travers une joint-venture avec un partenaire étranger; les autres terrains ont été cédés à l'une de ses filiales pour y construire des ensembles résidentiels (Huyền Trâm, 2019).

Dans les deux cas, ces sociétés ont engagé les travaux d'infrastructures prévus (mais non achevés en 2019). Toutefois, suite à la décision ( $\left.n^{\circ} 342 / T T g-V . I ~ d u ~ 07 / 03 / 2017\right)$ prise par le Premier ministre concernant la suspension temporaire des procédures de vente d'actifs fonciers publics (ou d'origine publique), les terrains récupérés par la ville dans le périmètre de la NZU de Thủ Thiêm et leur réaffectation à des investisseurs ont fait l'objet d'une inspection par différents services ministériels (VietnamNews, 2019). Les résultats de cette inspection révèlent plusieurs irrégularités, voire des allégations de corruption (Le Hong Viet, 2019). Pour certains observateurs, cela est peu étonnant, puisque la gestion des terres est depuis longtemps identifiée comme un domaine sensible sujet à la corruption au Vietnam, notamment lorsqu'il est question de montage impliquant des échanges terres-contre-infrastructure (Lê Hiệp, 2019). Dans ce cas précis c'est l'amplitude des irrégularités qui est remarquable.

D'un côté, les résultats des investigations rendues publiques ont révélé que le foncier acquis en l'échange de la construction de ces infrastructures a été évaluée à 26 millions $\mathrm{VND} / \mathrm{m}^{2}$ (soit $1130 \mathrm{USD} / \mathrm{m}^{2}$ ) (Hiếu Công, 2019). Pourtant, des économistes et des courtiers en immobilier estiment que la valeur marchande des terrains aurait aisément pu s'élever à 500 millions $\mathrm{VND} / \mathrm{m}^{2}$ (soit $22000 \mathrm{USD} / \mathrm{m}^{2}$ ) en raison des règles de construction dans la NZU de Thủ Thiêm qui permettent la réalisation de tours dépassant les 50 étages (Hiếu Công, 2019). De l'autre, les investigations ont mis en lumière le coût de la construction des routes réalisées par Đại Quang Minh. Il s'avère que $12 \mathrm{~km}$ de voirie ont été facturés 12182 milliards VND (environ 530 millions USD), 
soit environ 44 millions USD $/ \mathrm{km}$, ce qui fait de ces routes les "plus chères au monde " (Hiếu Công, 2018). Ces coûts de construction, approuvés par la municipalité (SGGP, 2019), semblent avoir été surestimés. De cet imbroglio, la commission d'inspection relevant du niveau central recommande de sanctionner la municipalité de Ho Chi Minh Ville et de réclamer près de 1,1 milliard de dollar US comme compensation en raison du manque à gagner pour le budget étatique dans la cession d'actifs fonciers publics (Le Anh, 2019).

La société CII est également dans la ligne de mire des services d'inspection gouvernementaux. L'irrégularité relevée dans le contrat entre cette société et la municipalité pointe une sous-évaluation des prix fonciers, mais surtout un clientélisme avéré. La société a été mandatée sans mise en compétition avec d'autres soumissionnaires. La commission d'inspection a relevé ici une irrégularité liée à l'attribution d'actifs fonciers publics. Ces derniers, au regard de la loi foncière de 2003, devant être mis aux enchères en vue de leur attribution au plus offrant (Tầm Nhìn, 2019). Au final les investigations ont permis de rendre compte d'informations qui, jusqu'à présent, étaient tenues confidentielles. En effet le contenu des contrats s'appuyant sur les échanges terres-contre-infrastructure est sensible et confidentiel, les coûts de construction des infrastructures peuvent être connus et révélés, mais ce sont le plus souvent les coûts de cession des terrains qui restent dissimulés.

L'opacité dans laquelle sont conduites les procédures d'ajustement de la planification urbaine comme la mise en œuvre de projets via le dispositif terres-contreinfrastructure mène d'un côté à une dégradation de la confiance des habitants envers les autorités et de l'autre à une dilapidation d'actifs publics dont la vente est supposée financer les infrastructures.

\section{Conclusion}

L'activité immobilière résidentielle dite "commerciale » dans le contexte vietnamien constitue un marqueur majeur du développement contemporain de Ho Chi Minh Ville. Cette activité est intense. Elle se caractérise par l'engagement d'un grand nombre d'opérations, par un important volume d'unités mises sur le marché, et par des formes urbaines inédites poussant à la verticalisation et à la densification. Le district 2 , avec en figure de proue la NUA de Thủ Thiêm, en constitue aujourd'hui la principale vitrine, suggérant ainsi que le gouvernement vietnamien s'appuie sur les processus d'urbanisation et sur les dynamiques des marchés immobilier et foncier urbains pour contribuer au développement économique du pays.

50 En premier lieu, cette intense activité se justifie d'abord par le fait qu'elle réponde à des besoins bien prégnants : loger une population urbaine croissante. En second lieu, ces opérations constituent des gains pour la municipalité : gains directs, à savoir des revenus fiscaux avec le paiement de redevances foncières lors de la cession de Droit d'Usage du Sol. Apparaissent également des gains indirects via la mise en place d'arrangements institutionnels incitant les acteurs économiques privés à produire des infrastructures et des équipements publics. Ho Chi Minh Ville, bien qu'étant la métropole la plus riche du Vietnam, dispose d'un budget insuffisant pour financer les infrastructures nécessaires à son développement territorial et économique et à son expansion. 
51 En raison des recettes limitées générées par la fiscalité immobilière et foncière, des alternatives ont été élaborées pour assurer la construction des infrastructures planifiées. Si des organisations internationales de développement comme la Banque mondiale encouragent le Vietnam à réformer sa fiscalité immobilière par l'introduction d'une taxe d'habitation (property tax) par exemple (Moser et Tideman, 2011), des solutions locales ont été adoptées. Pour la réalisation d'équipements publics et d'infrastructures, les partenariats impliquant le secteur public et le secteur privé (ou anciennement étatique) ont été engagés à travers le dispositif terres-contreinfrastructure. Ces partenariats prévoient qu'en l'échange de la construction d'un équipement, une société se voit attribuer des droits d'usage de terrains pour le développement d'opérations immobilières.

Sur le papier, ce modèle de financement et de production de la ville pourrait fonctionner. A la lumière du cas d'étude discuté dans cette contribution, force est de constater que ce modèle comporte non seulement des dérives, mais conduit à l'installation de pratiques de corruption et, dans la transition du plan vers le marché dans laquelle est le Vietnam, à un capitalisme de connivence. De plus, ce modèle ne fonctionne que si la ressource foncière est disponible (or il s'agit d'une ressource finie) et que celle-ci présente un potentiel de développement et de valorisation.

Le cas du district 2 et l'aménagement de la NZU de Thủ Thiêm ouvrent toutefois des perspectives salvatrices. Depuis 2017, alors que le gouvernement accentue sa lutte contre les pratiques de malversations et de corruption, des dispositifs de contrôle (commission d'inspection) ont révélé a posteriori que des actifs fonciers publics ont été bradés dans le cadre de l'aménagement de cette zone. Cela pose la question de la nécessité d'assurer la transparence de la mise en œuvre des projets où des actifs fonciers publics font l'objet d'échanges et également de créer des mécanismes de contrôle et d'évaluation indépendants. Jusqu'à présent, si la commission d'inspection parait remplir ce rôle, cette dernière intervient toutefois de manière curative et non préventive.

\section{BIBLIOGRAPHIE}

Bình Nguyên, 2018. Quand la carte de planification de Thủ Thiêm a-t-elle été perdue ? [Bản đồ quy hoạch Thủ Thiêm đã mất vào lúc nào?]. Zing News [En ligne], 05/05/2018. URL : https:// news.zing.vn/ban-do-quy-hoach-thu-thiem-da-mat-vao-luc-nao-post839872.html Boudreau J.A., Labbé D., 2011. Les nouvelles zones urbaines à Hanoi : ruptures et continuités avec la ville. Cahiers de géographie du Québec, vol. 55, n 154, p. 31-149.

Chatry I., 2019. A global view of sub-national governments in Asia: structure and finance. URL: https:// www.oecd-ilibrary.org/governance/fiscal-decentralisation-and-inclusive-growth-inasia_239e95db-en

David D., Gomez-Ibanez J.A., Thanh N.X., 2010. Ho Chi Minh City: The challenges of growth. Harvard Kennedy School. Ash Center for Democratic Governance and Innovation. Policy Dialogue Paper $n^{\circ} 2$ [En 
ligne]. URL: https://www.vn.undp.org/content/vietnam/en/home/library/poverty/ho-chiminh-city--the-challenges-of-growth.html

Do M.K., Zhang W., Zheng K., 2015. Reasons and Countermeasures of Status Quo of Vietnam Real Estate. In Zhang R., Zhang Z., Liu K., Zhang J. (ed), LISS 2013. Berlin, Springer, p. 1195-1200.

Douglass M., Huang L., 2007. Globalizing The City In Southeast Asia: Utopia On The Urban Edge. The Case Of Phu My Hung, Saigon. International Journal of Asian-Pacific Studies, vol. 3, n², p. 1-42.

DKRV, 2019. HCMC Residential Market In The First 8 Months Of 2019. Cushman \& Wakefield Real Estate Symposium Ho Chi Minh City, 12/09/2019.

Floater G., Dowling D., Chan D., et al., 2017. Global review of Finance for sustainable Urban Infrastructure [En ligne]. Coalition for Urban Transitions. URL: http://newclimateeconomy.net/ content/cities-working-papers

Gainsborough M., 2003. Changing political economy of Vietnam: the case of Ho Chi Minh City. Routledge, $200 \mathrm{p}$.

Gibert-Flutre M., 2018. La transition urbaine. In Treglode de B. (dir.), Histoire du Viêt Nam de la colonisation à nos jours. Paris, Éditions de la Sorbonne, p. 161-179.

Gillespie J., 2017. Transforming Land-Taking Disputes in Socialist Asia: Engaging an Authoritarian State. Law and Policy, vol. 39. p. 280-303.

Goldblum C., 2017. La transition urbaine dans l'ouverture à l'économie de marché. In Peyronnie K., Goldblum C., Sisoulath B., Transitions urbaines en Asie du Sud-Est : de la métropolisation émergente et de ses formes dérivées. Marseille, IRD éditions - IRASEC éditions, p. 351-350.

Ha H., Wong T.C., 1999. Economic reforms and the new master plan of Ho Chi Minh City, Vietnam: Implementation issues and policy recommendations. GeoJournal, vol. 49, n 3, p. 301-309.

Harms E., 2016. Luxury and Rubble. University of California Press, 304 p.

Hiếu Công, 2018. 1000 milliards VND/km, la route de Dai Quang Minh est la plus chère de la planète ["1.000 tỷ/ $\mathrm{km}$ thì đường Đại Quang Minh làm là đắt nhất hành tinh rồi”]. Zing News [En ligne], 09/05/2018. URL: https://news.zing.vn/1000-tykm-thi-duong-dai-quang-minh-lam-la-datnhat-hanhtinh-roi-post841072.html

Hiếu Công, 2019. Les enchères de terrains de Thủ Thiêm à partir de 11 millions VND $/ \mathrm{m}^{2}$ sont-elles trop bon marché ? [Đấu giá đất Thủ Thiêm khởi điểm từ 11 triệu đồng/m2 có quá rẻ?]. Zing News [En ligne], 18/09/2019. URL: https://news.zing.vn/dau-gia-dat-thu-thiem-khoi-diem-tu-11-trieudongm

HoREA (Ho Chi Minh City Real Estate Association), 2019. Rapport n²7/CV concernant la mise à jour des recommandations urgentes pour remédier aux plus gros goulots d'étranglement des entreprises et du marché immobilier ["V/v Báo cáo cập nhật các kiến nghị khẩn trương giải quyết những ách tắc lớn nhất của doanh nghiệp và thị trường bất động sản hiện nay”], 27/03/2019.

Huynh D., 2015a. The misuse of urban planning in Ho Chi Minh City. Habitat international, vol. 48, p. 11-19.

Huynh D., 2015b. Phu my hung new urban development in Ho Chi Minh City: Only a partial success of a broader landscape. International Journal of Sustainable Built Environment, vol. 4, $\mathrm{n}^{\circ} 1$, p. 125-135.

Huyền Trâm, 2019. Ho Chi Minh-Ville a approuvé l'investissement du projet de CII «bouclé » de près de 412 milliards de VND [TP.HCM duyệt đầu tư dự án của CII "vênh" gần 412 tỷ đồng]. BizLive [En 
ligne], 01/07/2019). URL: https://bizlive.vn/thoi-su/tphcm-duyet-dau-tu-du-an-cua-cii-venhgan-412-ty-dong-3512093.html

Kim A.M., 2008. Learning to be capitalists: entrepreneurs in Vietnam's transition economy. Oxford University Press, $224 \mathrm{p}$.

Kim A.M., 2009. Land takings in the private interest: Comparisons of urban land development controversies in the United States, China, and Vietnam. Cityscape, p. 19-31.

Labbé D., 2015. Once the Land Is Gone: Land Redevelopment and Livelihood Adaptations on the Outskirts of Hanoi, Vietnam. In Caballero-Anthony M., Barichello R. (ed.), Towards Balanced Growth: Alternative Development Models and Redistribution Mechanism. Nanyang Technological University Press, p. 148-180.

Labbé D., Musil C., 2014. Periurban Land Redevelopment in Vietnam under Market Socialism. Urban Studies, vol. 51, $\mathrm{n}^{\circ}$ 6, p. 1146-1161.

Le H.H., 2017. Vietnam's New Wave of SOE Equitization: Drivers and Implications [En ligne]. ISEAS, p. 2017-2057. URL: https://www.iseas.edu.sg/images/pdf/ISEAS_Perspective_2017_57.pdf

Le Hong Hiep, 2019. The Impact of Vietnam's Anti-corruption Campaign on the Real Estate Sector. ISEAS Perspective [En ligne], $\mathrm{n}^{\circ}$ 46, 7 p. URL: https://www.iseas.edu.sg/images/pdf/ ISEAS_Perspective_2019_46.pdf

Le Anh, 2019. Gov't Inspectorate proposes reclaiming investments in Thu Thiem project. Saigon Times [En ligne], 27/06/2019. URL: https://english.thesaigontimes.vn/69382/gov \%E2 \%80 \%99tinspectorate-proposes-reclaiming-investments-in-thu-thiem-project.html

Legoux M., 2011. Thu Thiem, vers un nouveau centre tertiaire majeur à Ho Chi Minh Ville, Vietnam Analyse du portage de projet et de la configuration d'acteurs. IFU-Université Paris-Est, mémoire de Master. $88 \mathrm{p}$.

Lê Hiệp, 2019. Auditeur général de l'État : district 1, des grandes pertes potentielles de terres, corruption [Tổng Kiểm toán Nhà nước: Quản l. đất đai tiềm ẩn lớn thất thoát, tham nhũng]. Thanh Niên [En ligne], 20/05/2019. URL: https://thanhnien.vn/thoi-su/tong-kiem-toan-nha-nuocquan-ly-dat-dai-tiem-an-lon-that-thoat-tham-nhung-1083741.html

Le Thanh Tung, 2016. La pratique de l'attraction de capitaux d'IDE dans le secteur immobilier à Ho Chi Minh Ville depuis l'accession à l'OMC. Prévisions et recommandations [Thực trạng thu hút vốn FDI vào bất động sản tại TP Hồ Chí Minh từ sau khi gia nhập WTO đến nay - Dự báo và khuyến nghị]. Review of Science \& Technology Development, vol. 19, n Q4 [en vietnamien].

Musil C., Perset M., 2015. Le financement des infrastructures de transport à Hô Chi Minh-Ville (ViêtNam) : les outils, les innovations et les défis, Working Paper $n^{\circ} 2$ du PADDI [En ligne]. URL: http:// www.codatu.org/wp-content/uploads/WP_PADDI_02_FR_low-quality.pdf

Musil C., Labbé D., Jacques O., 2019. Les zones urbaines «fantômes » de Hanoi (Vietnam). Éclairage sur le (dys)fonctionnement d'un marché immobilier émergent. Cybergeo : European Journal of Geography [En ligne]. URL: http://journals.openedition.org/cybergeo/31466 - DOI: https://doi.org/10.4000/cybergeo.31466

Morgan P.J., Trinh L.Q., 2016. Fiscal decentralization and local budget deficits in Viet Nam: an empirical analysis [En ligne]. ADBI Working Paper Series, $n^{\circ}$ 613. URL: https://www.adb.org/sites/default/ files/publication/210671/adbi-wp613.pdf

Moser B., Tideman N., 2011. Property and Land Taxes. In Gangadha P.S., Pham D.M., Engelschalk M. et al., Tax reform in Vietnam: Toward a more efficient and equitable system, p. 243-273. 
Murray P., Szelenyi I., 1988. L'anti-urbanisme des États socialistes. Annales de la recherche urbaine [En ligne], n 38, p. 23-30. DOI: http://doi.org/10.3406/aru.1988.1363

Nhật Minh, 2019. À quel point les projets de Dai Quang Minh à Thủ Thiêm étaient-ils mauvais ? [Các dự án của Đại Quang Minh ở Thủ Thiêm sai phạm như thế nào?]. Báo điện Tri Thức Trẻ [En ligne], 08/07/2019. URL: http://ttvn.toquoc.vn/kinh-doanh/cac-du-an-cua-dai-quang-minh-othu-thiem-sai-pham-nhu-the-nao-420198715511500.htm

Nhóm Phóng viên, 2018. Le Président du Comité populaire de Ho Chi Minh Ville : je m'excuse auprès des habitants de Thủ Thiêm [Chủ tịch UBND TP.HCM: Tôi chân thành xin lỗi người dân Thủ Thiêm]. Zing News [En ligne], 18/10/2018. URL : https://news.zing.vn/chu-tich-ubnd-tphcmtoi-chan-thanh-xin-loi-nguoi-dan-thu-thiem-post885256.html

Nguyen T.B., van der Krabben E., Musil C., Le D.A., 2018. 'Land for infrastructure' in Ho Chi Minh City: land-based financing of transportation improvement. International Planning Studies, vol. 23, $\mathrm{n}^{\circ} 3$, p. 310-326.

Novaland, 2019. Investor Presentation. August 2019.

PADDI, 2010. Atelier méthodologique mise en œuvre de la planification urbaine à HCMV. Les Livrets du Centre de prospective et d'études urbaines - PADDI, 14-22 Juin 2010.

PADDI et Agence d'Urbanisme de l'Agglomération Lyonnaise, 2012. Comment rendre la planification urbaine au Viêt-Nam plus efficace ? In Aderef, Forum économique et financier, $g^{e ̀ m e}$ session pour une urbanisation durable à la périphérie des villes vietnamiennes.

Pandolfi L., 2001. Une terre sans prix : Réforme foncière et urbanisation au Viêt-Nam, Hà Nội, 1986-2000. Thèse de doctorat, Université de Paris 8 Vincennes Saint-Denis.

Pham Thai Son, 2014. Property Market and the Development of Vietnamese Metropolis: Analysis of Land Use and Spatial Distribution of Housing Projects in Ho Chi Minh City, Conference paper ISCP 2014.

PWC, 2019. Emerging Trends in Real Estate, Asia Pacific 2019. URL: https://www.pwc.com/sg/en/ publications/assets/emerging-trends-real-estate-asia-pacific-2019.pdf

Quertamp F., Pandolfi L., Petibon L., 2014. Faire la ville : Lecture croisée des méthodes et outils de l'urbanisme en France et au Viet-Nam. Capitalisation des expériences de la coopération décentralisée PADDI-IMV, $63 \mathrm{p}$.

Quertamp F., 2017. Hô Chi Minh Ville, une planification centralisée à l'épreuve des grands projets. In Peyronnie K., Goldblum C., Sisoulath B., Transitions urbaines en Asie du Sud-Est : de la métropolisation émergente et de ses formes dérivées. IRD éditions - IRASEC éditions, p. 81-108.

Shatkin G., 2016. The real estate turn in policy and planning: Land monetization and the political economy of peri-urbanization in Asia. Cities [En ligne], $n^{\circ}$ 53, p. 141-149. URL: http://dx.doi.org/ 10.1016/j.cities.2015.11.015 - DOI: https://doi.org/10.1016/j.cities.2015.11.015

Shatkin G., 2017. Cities for Profit: The Real Estate Turn in Asia's Urban Politics. Ithaca, Cornell University Press, 296 p.

Tầm Nhìn, 2019. Qu'y a-t-il de mal à ce que la société CII «reste» dans la nouvelle zone urbaine de Thủ Thiêm? [Công ty CII "dính" sai phạm gì tại khu đô thị mới Thủ Thiêm?]. Tầm Nhìn [En ligne], 04/07/2019. URL: https://tamnhin.net.vn/cong-ty-cii-dinh-sai-pham-gi-tai-khu-do-thi-moi-thuthiem-73227.html

Tiếng Dân, 2018. Perdu à Thủ Thiêm ? [Mất gì ở Thủ Thiêm?]. Tiếng Dân [En ligne], 09/05/2018. URL: https://baotiengdan.com/2018/05/10/mat-gi-o-thu-thiem/ 
Thanh Niên, 2018. Comment le Comité Populaire de Ho Chi Minh Ville a ajusté la planification spatiale de Thủ Thiêm" [UBND TP.HCM đã điều chỉnh quy hoạch Thủ Thiêm như thế nào?]. Thanh Niên [En ligne], 4/05/2018. URL: https://thanhnien.vn/thoi-su/ubnd-tphcm-da-dieu-chinh-quyhoach-thu-thiem-nhu-the-nao-959260.html

Thanh tra Chính phủ (Inspection du gouvernement), 2018. Thông báo [Rapport] 1483/TB-TTCP [En ligne]. 04/09/2018. URL: http://www.thanhtra.gov.vn/ct/news/Lists/KetLuanThanhTra/ View_Detail.aspx?ItemID=104

Truitt A.J., 2013. Dreaming of Money in Ho Chi Minh City. University of Washington Press, 224 p. Vasavakul T., 2019, Vietnam: A Pathway from State Socialism. Cambridge University Press, 86 p. VietnamNews, 2019. Government Inspectorate report critical of HCM City handling of Thủ Thiêm. VietnamNews [En ligne], 28/06/2019. URL: https://vietnamnews.vn/society/521926/governmentinspectorate-report-critical-of-hcm-city-handling-of-thu-thiem.html

World Bank, 2013. Assessment of the financing framework for municipal infrastructure in Vietnam. Final Report [En ligne]. URL: http://documents1.worldbank.org/curated/en/224031468126286710/ pdf/ACS59190Vietna0tnameseofinalomerged.pdf

World Bank et Ministry of Planning and Investment, 2016. Vietnam 2035: Toward Prosperity, Creativity, Equity, and Democracy [En ligne]. URL: http://vids.mpi.gov.vn/Includes/NewsDetail/ 12_2016/dt_11220161028_vn2035english.pdf

World Bank, 2019. The Socialist Republic of Vietnam for the first Ho Chi Minh City Development Policy Operation, Rapport n ${ }^{\circ} 136241-V N$ [En ligne]. URL: http://documents1.worldbank.org/curated/en/ 732191558317696718/pdf/Vietnam-First-Ho-Chi-Minh-City-Development-Policy-OperationProject.pdf

Waibel M, 2006. The Production of Urban Space in Vietnam's Metropolis in the course of Transition: Internationalization, Polarization and Newly Emerging Lifestyles in Vietnamese Society. Trialog, vol. 89, nº 2, p. 43- 48.

\section{NOTES}

1. Il s'agit d'une série de réformes qui ont transformé à partir du début des années 1980 le système socio-économique du Vietnam pour le placer sur la voie de l'« économie de marché à orientation socialiste » sous le contrôle du Parti communiste vietnamien.

2. Pour exemple, concernant le seul secteur du transport public, un volume d'investissement de plus de 18 milliards USD est budgété d'ici à 2030 pour la construction d'un vaste réseau comprenant entre autres 8 lignes de métro. En 2020, une seule ligne de métro est en cours de construction.

3. Dans le contexte vietnamien, il s'agit d'une privatisation partielle : l'État ouvre le capital des entreprises publiques tout en demeurant un actionnaire. Ces entreprises sont depuis censées fonctionner comme des entreprises privées avec des objectifs de compétitivité et de rentabilité (Le, 2017).

4. Concernant les projets immobiliers ces droits sont valables entre 50 et 70 ans selon le type d'opération immobilière.

5. La loi foncière de 1993 stipule que les droits d'usages du sol, et non pas le sol lui-même, peuvent être transmis en héritage, loués, échangés, hypothéqués et cédés à titre onéreux.

6. Par exemple : taxes d'enregistrement des DUS (0.5\% de la valeur des terrains), taxes d'usage de terrains non-agricole (de $0.03 \%$ à $0.2 \%$ de la valeur des terrains), taxe sur le transfert des DUS ( $2 \%$ 
de la valeur des terrains) et le coût des DUS pour une durée limitée à 50 ans dans le cas de projets d'immobiliers (pour une discussion plus détaillée, voir Moser et Tideman, 2016).

7. Le PIB par habitant au Vietnam en 2018 était de 2525 USD contre 6800 USD pour Ho Chi Minh Ville (World Bank, 2019)

8. La détermination des prix fonciers est complexe au Vietnam en raison de la coexistence de deux prix : le prix du marché et celui fixé par l'administration. Les prix fonciers fixés par l'administration sont souvent de $50 \%$ à $70 \%$ sous ceux du marché.

\section{RÉSUMÉS}

À partir du cas de l'aménagement du district 2 et de la Nouvelle Zone Urbaine de Thủ Thiêm cet article explore le contexte au sein duquel prend place l'accroissement du rôle des acteurs privés de la promotion immobilière dans la production métropolitaine de Ho Chi Minh Ville et ses effets sur la fabrique des nouveaux territoires urbains. Alors que le marché immobilier résidentiel de la métropole du sud du Vietnam est l'un des plus dynamiques en Asie du Sud-Est, les pouvoirs publics s'appuient sur les acteurs de ce secteur afin de produire les infrastructures nécessaires au développement territorial et économique de la métropole. Malgré la mise en place de garde-fous, des dérives apparaissent et participent à la dilapidation d'actifs publics dont la vente est supposée financer l'urbanisation en cours.

Using the case of the district 2 and Thủ Thiêm New Urban Area, this article explores the growing importance of private property developers in Ho Chi Minh City and its effects on the production of new urban territories. While the residential property market of Ho Chi Minh City is among the most dynamic in Southeast Asia, the local government relies on private developers and investors to produce the infrastructure needed by the metropolis and to ensure its economic and territorial growth. Despite existing safeguards, official rules and regulations are bypassed, contributing to the dilapidation of public assets meant to finance the ongoing urbanization.

\section{INDEX}

Keywords : real estate, land, urban policy, Ho Chi Minh City, Vietnam

Mots-clés : immobilier, foncier, politique urbaine, Ho Chi Minh Ville, Vietnam

Thèmes : Sur le Champ - Sur le Terrain

\section{AUTEURS}

\section{CLÉMENT MUSIL}

Clément Musil, musil.clement@gmail.com, est chercheur associé à l'UMR AUSser 3329 et à

l'Université de Montréal (Canada). Il a notamment publié :

- Musil C., 2020. Hong Kong's “Rail-plus-Property" development, a model for financing public transport in South-East Asian developing cities? In Rengarajam P., Bing Z., Rabé P. (ed.), Future Challenges of Cities. IIAS: Leiden (Netherlands), Amsterdam University Press. 
- Musil C., Labbé D., Jacques, O., 2019. Les zones urbaines «fantômes » de Hanoi (Vietnam). Éclairage sur le (dys)fonctionnement d'un marché immobilier émergent. Cybergéo: European Journal of Geography [En ligne]. URL: https://journals.openedition.org/cybergeo/31466 - DOI: https://doi.org/10.4000/cybergeo.31466

- Nguyen T. B., Van Der Krabben E., Musil C., Le A.D., 2018. "Land for Infrastructure” in Ho Chi Minh City: Land-based Financing of Transportation Improvement. International Planning Studies [En ligne], vol. 23, n 3, p. 310-326. URL: https://www.tandfonline.com/doi/abs/ 10.1080/13563475.2018.1477581 - DOI: https://doi.org/10.1080/13563475.2018.1477581

\section{DANIELLE LABBÉ}

Danielle Labbé, danielle.labbe@umontreal.ca, est Professeure associée à l'École d'urbanisme et d'architecture de paysage et titulaire d'une Chaire de recherche à l'Université de Montréal, Canada. Elle a récemment publié :

- Labbé D., Sorensen A. (ed.), 2020. Handbook of Megacities and Megacity-Regions. Cheltenham (UK), Edward Elgar Publishing.

- Petter A.M., Labbé D., Lizzaralde G., Goulet J., 2020. City profile: Canaan, Haiti - A new post disaster city. Cities, $\mathrm{n}^{\circ}$ 104. DOI: https://doi.org/10.1016/j.cities.2020.102805

- Florez-Bossio C., Forde J., Labbé D., 2019. Adaptive Capacity in Urban Areas of Developing Countries. Climatic Change, $\mathrm{n}^{\circ}$ 157, p. 279-297.

\section{VU NGOC KHANH}

Vu Ngoc Khanh, nvu@swin.edu.au, est doctorant au Centre for Urban Transitions, Swinburne University of Technology, Melbourne (Australie). 